\title{
Speech Enhancement by Non-Stationary Tonal Disturbance Cancellation using Subband Zero Crossing Measures
}

\author{
Nils Westerlund \\ nils.westerlund@bth.se
}

\author{
Mattias Dahl \\ mattias.dahl@bth.se
}

\author{
Ingvar Claesson \\ ingvar.claesson@bth.se
}

\author{
School of Engineering \\ Applied Signal Processing Group \\ Blekinge Institute of Technology \\ Ronneby, Sweden
}

May, 2004

\begin{abstract}
A time domain based method for rejection of tonal disturbances in speech signals is presented. Tonal disturbances in personal communication arise in a variety of situations such as environmental sounds from rotating machinery, acoustic feedback in PA-systems or hearing aids and internal circuitry of some personal communication devices. The method is based on the zero crossings of subband signals. After splitting the disturbed signal into subbands, the distances between zero crossings in the subband signals are measured. A low distance spread indicates that a tonal component is dominating the subband signal. This distance spread is then used to steer a gain function that adaptively and continuously attenuates subbands in which tonal components are dominant. The method has proven to be effective on stationary as well as non-stationary disturbances with reasonable amount of speech signal distortion.
\end{abstract}

\section{Introduction}

Tonal disturbances in speech communication may arise in a variety of situations. Surrounding sound sources such as sirens or rotating machinery may emit tonal sounds; the internal circuitry of speech communication equipment like cellular phones may add tonal components to the transmitted speech signal [1]. A very common situation where high amplitude tonal disturbances are encountered is when acoustic feedback is induced in a PA-system or in hearing aids. Another typical example of a tonal disturbance is the 50 or $60 \mathrm{~Hz}$ power line hum. In addition, detection of tonal sounds may be desirable in, for example, analogue modem communication or dual-tone multifrequency (DTMF) telephone systems.

Intuitively, a fixed notch filter tuned to the frequency of interest may be used to eliminate these kind of disturbances. A very narrow notch is usually desired in order to filter out the interference without distorting the signal. Problems arise when the frequency of the interference is not precisely known or if the frequency is non-stationary. If the notch is very narrow, it may not be located exactly over the interference frequency. In addition, estimating the frequency of several moving tones embedded in a signal and possibly broad-band noise may result in high computational complexity [2], [3].

Other algorithms used to adaptively determine parameters like frequency, phase or magnitude are generally called Adaptive Line Enhancers (ALE's). Traditionally, ALE's have been realized using FIR filters [4] but IIR filter solutions have also been investigated $[5],[6]$. 
Another method for suppression of non-stationary tonal interferences is transform median filtering. Since a sinusoid appears as a large and narrow frequency signal component, a median filter may be employed in order to reduce this component in frequency domain [7].

The Zero Crossing (ZC) measure is a non-linear measure that has traditionally been used for analysis, segmentation and recognition of speech sounds. The ZC measure has proven to be well suited to digital signal processing [8], [9].

The research described in this paper was conducted in order to explore the possibility of using a ZC based measure in order to detect and attenuate tonal disturbances in speech signals. In the proposed method, the sample distance between zero crossings in subband signals are measured. The distance spread is utilized over time to calculate the upper and lower spread and the distance between these spread estimates controls a subband gain function that is used to weigh the subbands proportionally to the $\mathrm{ZC}$ distance spread.

\section{Problem Formulation Method}

Assume a discrete time speech signal $s(n)$ and a tonal signal $w(n)$ considered as being a disturbance. The corrupted speech signal $x(n)$ can then be written as $x(n)=s(n)+w(n)$. By filtering the input signal $x(n)$ using a bank of $K$ bandpass filters, $h_{k}(n)$, the signal is divided into $K$ subbands, each denoted by $x_{k}(n)$ where $k$ is the subband index. This filtering operation can be written in time domain as $x_{k}(n)=x(n) * h_{k}(n)$ where $*$ is the convolution operator. In the ideal case, the original signal can then be described as

$$
x(n)=\sum_{k=0}^{K-1} x_{k}(n)=\sum_{k=0}^{K-1} s_{k}(n)+w_{k}(n)
$$

i.e. $x_{k}(n)=s_{k}(n)+w_{k}(n)$ where $s_{k}(n)$ is the speech part in subband $k$ and $w_{k}(n)$ is the tonal disturbance in the same subband.

The subband ZC measure $Z_{k}(n)$ is then calculated as

$$
Z_{k}(n)=\frac{\left|\operatorname{sgn}\left\{x_{k}(n)\right\}-\operatorname{sgn}\left\{x_{k}(n-1)\right\}\right|}{2}
$$

where the sign function $\operatorname{sgn}\{\cdot\}$ is defined as

$$
\operatorname{sgn}\left\{x_{k}(n)\right\}=\left\{\begin{array}{l}
+1 \text { if } x_{k}(n) \geqslant 0 \\
-1 \text { if } x_{k}(n)<0
\end{array}\right.
$$

Note that $Z_{k}(n)=1$ if a change in sign has occurred between sample $n$ and $n+1$ in $x_{k}(n)$ and $Z_{k}(n)=0$ otherwise.

When a subband zero crossing occurs the sample index $n$ is stored in a vector $Z_{k, i x}(l), l=\left[0 \ldots M_{k}-\right.$ $1]$, where $Z_{k, i x}(0)$ is the most recent zero crossing sample index. The vector $Z_{k, i x}(l)$ is continuously updated in a first-in-first-out manner (FIFO).

The distance in samples between two zero crossings in $x_{k}(n)$ is then given by

$$
D_{k, z c}(n)=Z_{k, i x}(0)-Z_{k, i x}\left(M_{k}-1\right)
$$

where $M_{k}$ is used to control between which zero crossings the distance is calculated.

Equation (4) results in a vector with a certain spread, or envelope. However, a constant spread of $D_{k, z c}$ is indicating that a tonal component is dominant in the subband. Hence, means are needed to track the spread of $D_{k, z c}$. The upper and lower zero crossing distance spread, $D_{k, u}(n)$ and $D_{k, l}(n)$ respectively, is then calculated as

$$
\begin{aligned}
D_{k, u}(n)= \begin{cases}D_{k, z c}(n) & \text { if } \mathcal{A} \\
\alpha_{k, 1} D_{k, u}(n-1)-\alpha_{k, 2} D_{k, z c}(n) & \text { if } \mathcal{B}\end{cases} \\
\\
\mathcal{A}: \quad D_{k, z c}(n) \geqslant D_{k, u}(n-1) \\
\mathcal{B}: \quad D_{k, z c}(n)<D_{k, u}(n-1)
\end{aligned}
$$

and

$D_{k, l}(n)= \begin{cases}D_{k, z c}(n) & \text { if } \mathcal{C} \\ \beta_{k, 1} D_{k, l}(n-1)+\beta_{k, 2} D_{k, z c}(n) & \text { if } \mathcal{D}\end{cases}$
$\mathcal{C}: \quad D_{k, z c}(n) \leqslant D_{k, l}(n-1)$

$\mathcal{D}: \quad D_{k, z c}(n)>D_{k, l}(n-1)$ 
Table 1: Parameter Selection

\begin{tabular}{l||ll}
\hline Parameter & Value \\
\hline \hline$M_{k}$ & $10 k \quad \forall k$ \\
\hline$\alpha_{k, 1}, \alpha_{k, 2}$ & $\left(1-10^{-4}\right), 10^{-4} \quad \forall k$ \\
\hline$\beta_{k, 1}, \beta_{k, 2}$ & $\left(1+10^{-4}\right), 10^{-4} \quad \forall k$ \\
\hline$\gamma_{k}, \delta_{k}$ & $0.99,1.0005 \quad \forall k$ & \\
\hline$L_{k, u}, L_{k, l}$ & $1,0.01 \quad \forall k$ & \\
\hline
\end{tabular}

In (5) and (6), $\alpha_{k, 1}, \alpha_{k, 2}, \beta_{k, 1}$ and $\beta_{k, 2}$ are parameters controlling the upper and lower spread tracking characteristics of the functions $D_{k, u}(n)$ and $D_{k, l}(n)$.

Finally, the zero crossing spread is given by

$$
S_{k}(n)=\left|D_{k, u}(n)-D_{k, l}(n)\right|
$$

The subband gain function $G_{k}(n)$ based on the spread measure $S_{k}(n)$ is now calculated as

$$
\begin{gathered}
G_{k}(n)=\left\{\begin{array}{l}
\gamma_{k} \cdot G_{k}(n-1) \text { if } \mathcal{E} \\
\delta_{k} \cdot G_{k}(n-1) \text { if } \mathcal{F}
\end{array}\right. \\
\mathcal{E}: \quad 0 \leqslant S_{k}(n) \leqslant 1, \quad G_{k}(n-1)>L_{k, l} \\
\mathcal{F}: \quad S_{k}(n)>1, \quad G_{k}(n-1)<L_{k, u}
\end{gathered}
$$

In equation (8), $\gamma_{k}$ and $\delta_{k}$ are parameters controlling the positive and negative slope of the gain function and $L_{k, u}$ and $L_{k, l}$ are the upper and lower limits of $G_{k}(n)$, respectively. In addition, if $G_{k}(n)$ exceeds $L_{k, u}$ or fall below $L_{k, l}$ it is truncated to the corresponding value. Note that $\gamma_{k}<1$ and $\delta_{k}>1$.

\section{Evaluation}

For evaluation purposes a linearly spaced 16-band FIR filter bank (128 tap filter length) was created. A speech signal was recorded, properly bandlimited and sampled at $F_{s}=8 \mathrm{kHz}$. Two sine functions were added to this speech signal: One stationary $2 \mathrm{kHz}$ tone and one non-stationary sinusoidal sweep. The disturbed signal was divided into subbands and run through the algorithm on a sample by sample basis. The parameter settings are shown in Tab. 1.
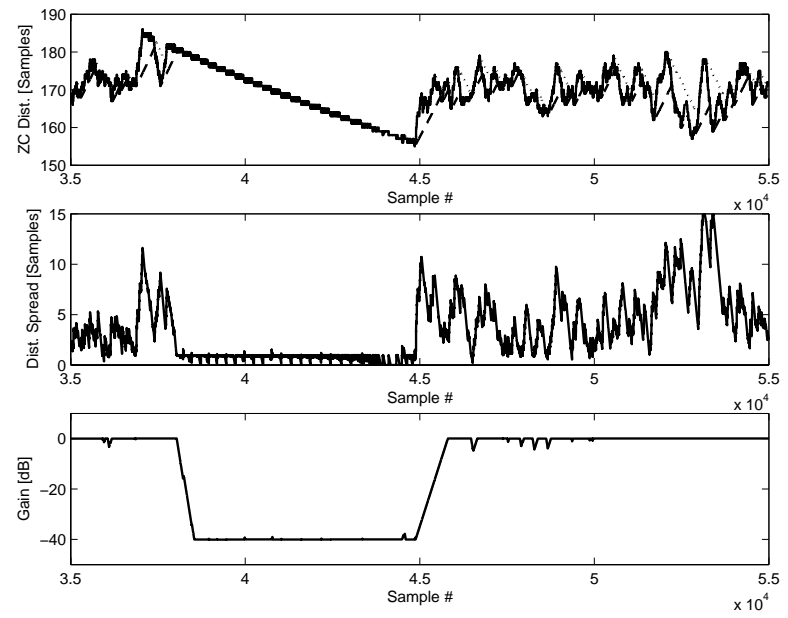

Figure 1: Results from data run with speech distorted by a swept sine. The plots show data from subband $k=8$. (Upper plot) Solid line - Zero crossing distances, $D_{k, z c}(n)$, Dotted line - Upper spread tracking function $D_{k, u}(n)$, Dashed line - Lower spread tracking function $D_{k, l}(n)$. (Mid plot) Zero crossing spread $S_{k}(n)$. (Lower plot) Gain function $G_{k}(n)$.

In Fig. 1 data from different steps in the algorithm is shown. The upper plot illustrates the impact of an incoming tone into the subband, in this case subband $k=8$. When the tone enters the subband the ZC measure stabilizes, i.e. the zero crossing spread decreases to zero or one sample. This is illustrated in the mid plot. As long as the spread is greater than unity the resulting gain function (shown in Fig. 1, lowermost plot) is increasing until it reaches the upper limit given by $L_{k, u}$. However, as previously stated, when a tonal disturbance enters the subband the zero crossing distance spread decreases, resulting in a rapid decrease of the gain function. This decrease is limited by $L_{k, l}$. In effect, the gain function will attenuate the incoming tone as long as it is present in the subband at hand. When only speech is present in the subband, the gain function will increase to unity again. 

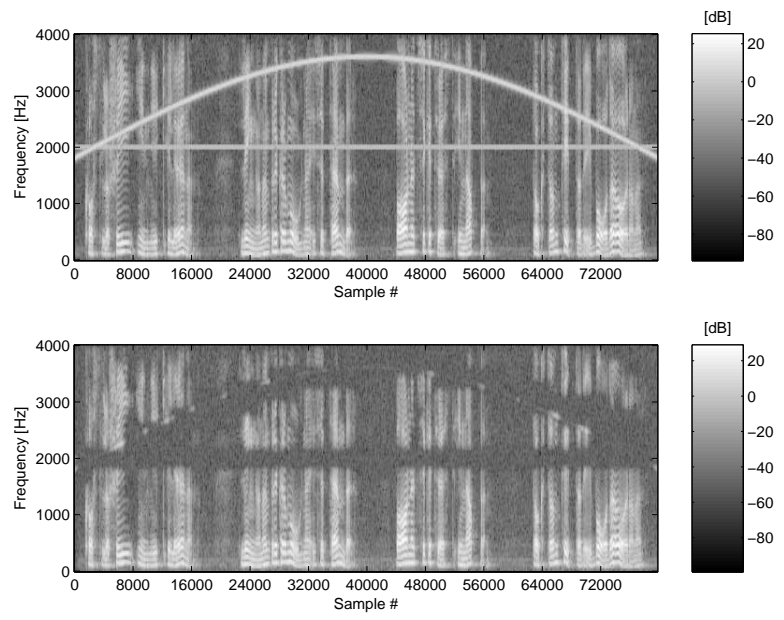

Figure 2: (Upper plot) Spectrogram of input data with speech distorted by a stationary and a nonstationary sinusoid. (Lower plot) Spectrogram of enhanced output data.

\section{Results}

Spectrograms of the algorithm input and output are shown in Fig. 2. As one can see both sinusoids are efficiently attenuated throughout the data sequence.

\section{Conclusions}

Tonal disturbances are quite common in personal communication. Sirens or machines may emit tonal sounds; the internal circuitry of speech communication equipment like cellular phones may add tonal components to the transmitted speech signal and the performance of an ordinary PA-system may be severely degraded by acoustic feedback. This urges for efficient speech enhancement algorithms for rejection of non-stationary disturbances.

A modern speech enhancement algorithm must be flexible yet robust, easily implemented yet computationally efficient and, in addition, it must be versatile and applicable to many different noise situations. The ZC measure based method described in this paper fulfills all of these criteria.

The initial study of the proposed method reveals that both stationary and non-stationary tonal disturbances are suppressed efficiently with a small amount of remaining speech distortion. Care must be taken in order to choose the parameter settings correctly but a fair basic default setting was presented in this paper.

\section{References}

[1] A. Nilsson I. Claesson, "Gsm tdma frame rate internal active noise cancellation," International Journal of Acoustics and Vibration, vol. 8, no. 3, 2003.

[2] J. R. Glover, "Adaptive noise canceling applied to sinusoidal interferences," IEEE Trans. Acoust. Speech and Sig. Proc., vol. ASSP-25, no. 6, pp. 484-490, December 1977.

[3] J. Okello, S. Arita, Y. Itoh, Y. Fukui, and M. Kobayasi, "An adaptive notch filter for eliminating multiple sinusoids with reduced bias," in Proc. ISCAS, 2000, vol. III, pp. 551-554.

[4] B. Widrow and S. D. Stearns, Adaptive Signal Processing, Prentice-Hall, New Jersey, 1985.

[5] M. H. Hayes, Statistical Digital Signal Processing and Modeling, Wiley, 1996.

[6] T. Kwan and K. Martin, "Adaptive detection and enhancement of multiple sinusoids using a cascade iir filter," IEEE Trans. Circuits and Systems, vol. 36, no. 7, pp. 937-947, July 1989.

[7] T. Kasparis, "Suppression of nonstationary sinusoidal interference using transform domain median filtering," IEEE Electronics Letter, vol. 29, no. 2, pp. 176-178, January 1993.

[8] J. R. Deller Jr., J. G. Proakis, and J. H. L. Hansen, Discrete time processing of speech signals, Macmillan Publishing Company, 1993.

[9] M. R. Ito and R. W. Donaldson, "Zero-crossing measurements for analysis and recognition of speech sounds," IEEE Trans. on Audio and Electroacoustics, vol. AU-19, no. 3, pp. 235-242, September 1971. 